\title{
Original
}

\section{TMJ Degenerative Changes in SAMP3 Mice by Occlusal Disharmony and Aging}

\author{
Abir Elshawi' ${ }^{1)}$, Noriko Wakamatsu' ${ }^{1)}$,Mitsuo Iinuma ${ }^{1)}$, Motohiko Nagayama ${ }^{2)}$ and Yasuo Tamura ${ }^{1)}$ \\ 1) Department of Pediatric Dentistry, Division of Oral Structure, Function and Development,Asahi University School of \\ Dentistry, Gifu, Japan \\ 2) Department of Oral Pathology, Division of Oral Pathogenesis and Disease Control, Asahi University School of Dentistry, Gifu, \\ Japan
}

(Accepted for publication, May 25, 2012)

\begin{abstract}
Aging and mechanical loading by occlusal disharmony are critical and etiological factors for the onset of temporomandibular joint disorders (TMDs). Senescence-accelerated mouse prone 3 (SAMP3) is one of the animal models for studying age-related degenerative changes of various tissues and organs. To evaluate the influences of both the aging and/or the mechanical loading induced by occlusal disharmony on the SAMP3 TMJ condyle, histological and immunohistochemical analyses were performed.

Fifty-five, 4-week-old SAMP3 mice were divided into two groups as an experimental and control. In the experimental group, the maxillary molars were trimmed out from the occlusal plane, which also served as mechanical force loading on the condylar surface. Then the condyles were removed and proceeded for histological and immunohistochemical analysis. The subchondral bone ossification was also evaluated by micro-focusing computed tomography (micro-CT) analysis.

The results indicated that induced occlusal disharmony in an aging background promotes TMD, including osteoarthritis (OA) like disease. OA-like TMJ lesions in the SAMP3 mice, accompanied by mandibular condylar cartilage degradation, were characterized by loss of proteoglycans (PGs) and changes of the localization of the extracellular matrix, such as collagen type I, II and MMP13. Changes in the microarchitecture of the condyle were also caused under OA-like lesion. Disrupted Wnt/ $\beta$-catenin signaling by means of immunohistochemical analysis of $\beta$-catenin contributes to this irreversible cartilage tissue damage.

The present study indicates that the experimentally induced occlusal disharmony (under the age-related background) promotes the TMJ OA accompanied by the alteration to components of the extracellular matrix, and $\mathrm{Wnt} / \beta$-catenin signaling disorder contributes to these irreversible cartilage tissue damage.
\end{abstract}

Key words:TMJ, Mandibular condyle, Aging, SAMP3 mice, Osteoarthritis

\section{Introduction}

Aging and mechanical loading by occlusal disharmony following teeth extraction or prosthodontic dental care are two critical and etiological risk factors for the onset of temporomandibular joint disorders (TMDs) ${ }^{1-4)}$. Senescence-accelerated mouse prone 3 (SAMP3), one of the senescence-accelerated mouse (SAM) strains, is a well-known animal model for the studying age-related degenerative changes of various tissues and organs, including the articular cartilage of the temporomandibular joint (TMJ). These mouse strains are also characterized by a shorter lifespan and the early manifestation of various signs of senescence, such as altered

Correspondence to:Dr. Motohiko Nagayama. Department of Oral Pathology, Division of Oral Pathogenesis and Disease Control, Asahi University School of Dentistry,1851-1, Hozumi, Mizuho-City, Gifu 501-0296, Japan. Tel: +81-58-329-1427, Fax: +81-58-329-1427, E-mail: moto@dent.asahi-u.ac.jp physical activity and loss of hair ${ }^{5)}$. The SAMP3 strain shows not only an early and high incidence of severe degeneration in the TMJ, but these changes start at three months of age ${ }^{6}$. One of the severe pathological changes in TMD is a destruction of the joint cartilage, known as osteoarthritis (OA), which is characterized by degradation and erosion along the surface of articular cartilage ${ }^{7)}$ and results in changes to the subchondral bone structure ${ }^{8)}$.

Articular chondrocytes, the predominant cell type present in articular cartilage, are responsible for producing and maintaining the extracellular matrix (ECM), which supports the appropriate biomechanical function of their tissue. This is mainly composed of fibrous collagens and proteoglycans (PGs) ${ }^{9}$. Matrix metalloproteinases (MMPs) are considered to be the key enzymes in the degradation of these ECM constituents ${ }^{10,11)}$. In particular, MMP-13 is one of the most important MMPs in cartilage 
remodeling, exhibiting a substrate preference for the cartilagespecific type II collagen ${ }^{12}$, a major collagen in cartilage matrix ${ }^{13}$, 14). Phenotypic changes seen in TMD-OA are often accompanied by ECM degradation, which eventually results in cartilage destruction, joint deformity and dysfunction of the TMJ.

Chondrocyte function in articular cartilage is regulated by a variety of growth factors, including the $\mathrm{Wnt} / \beta$-catenin signaling molecules, which play a critical role in chondrocytic proliferation, differentiation, and apoptosis. $\beta$-catenin is a key molecule in the canonical Wnt signaling pathway and is crucial during the multiple steps of chondrocyte formation, maturation and joint remodeling via transcription factors, such as T-cell factor and lymphoid enhancer factor, TCF/LEF ${ }^{15}{ }^{16}$. This canonical signaling functions to maintain the cartilage matrix. Furthermore, it is unique to differentiating chondrocytes, with cells exhibiting a very limited mitotic activity, a slow rate of matrix synthesis, degradation at the pre-hypertrophic layer, and a failure to progress fully into a terminally differentiated phenotype, as displayed by the presence of localized $\beta$-catenin in the nucleus of chondrocytes in the prehypertrophic layer ${ }^{17)}$. However, the mechanism of TMD-OA pathogenesis still remains undefined ${ }^{18)}$.

In this study, to evaluate the influence of the aging and the mechanical loading on the SAMP3 TMJ condyle, histological and immunohistological analyses were performed.

\section{Materials and Methods}

\section{Animals}

SAMP3 mice used in this study was kindly donated from Dr. Masanori Hosokawa, MD, PhD, an animal holder and were introduced to Asahi University after check as a specific pathogenfree and mating under the conventional condition at the Breeder (SAMP3/SlcIdr, Japan SLC Inc., Shizuoka, Japan). Fifty-five, 4week-old SAMP3 mice (30 males and 25 females) were divided into two groups: experimental and control (no treatment). In the experimental group, the maxillary molars on both sides were trimmed out of occlusion to an approximate $1 \mathrm{~mm}$ loss of the level of the occlusal plane using a handy micro motor (NE77-Yos, Yoshida, Tokyo, Japan); this also served as mechanical loading on the condyle. The mice, weighing between $25 \mathrm{~g}$ to $30 \mathrm{~g}$, were sacrificed at different stages: 3, 6, and 9 months. All animal experiments were performed according to the guidelines for animal care of the Asahi University.

\section{Tissue preparation}

For histological and immunohistochemical analyses, the TMJ from each mouse was dissected and fixed in $4 \%$ paraformaldehyde (PFA) for $24 \mathrm{~h}$ at $4{ }^{\circ} \mathrm{C}$. The specimens were rinsed twice with $1 \%$ PBS for 10 min, decalcified in $10 \%$ ethylenediaminetetraacetic acid (EDTA) solution at pH 7.2-7.4 for 4 weeks, and dehydrated in graded ethanol. Finally, the specimens were passed through xylene and embedded in paraffin. Serial $3 \mu \mathrm{m}$-thick sections of the mandibular condyle were sliced parallel to the parasagittal plane in an anterior-posterior direction.

\section{Histological and immunohistochemical analysis}

Serial sections of the TMJ were stained with the hematoxylin and eosin (HE) for histological observation, or with $0.1 \%$ Safranin1 st green staining. For immunohistochemistry, deparaffinized sections were treated with $0.3 \%$ hydrogen peroxide for $2 \mathrm{~h}$ at room temperature to block endogenous peroxidase activity. Prior to primary antibody reaction, sections were enzymatically digested for $30 \mathrm{~min}$ at $37^{\circ} \mathrm{C}$ with hyaluronidase for unmasking of antigen (EC: 4.2.2.1, Amano, Tokyo). Polyclonal antibodies against type I collagen (rabbit anti-type I collagen, AB765P, Millipore, US), type II collagen (rabbit anti-bovine type II collagen, LB-1297, LSL, US), matrix metalloproteinase-13 (MMP-13, goat antiMMP-13 AB8120, Millipore, US) or $\beta$-catenin (rabbit anti- $\beta$ catenin, Takara Bio, Kyoto, Japan) were listed and incubated for $2 \mathrm{~h}$ at room temperature, and then incubated with horseradish peroxidase-labeled secondary antibody to react with primary antibody. Color detection was performed with 3,3'diaminobentidine (DAB) substrate solution-brown chromogen reaction.

\section{Micro-CT}

A three-dimensional morphometric analysis of the subchondral bone of the mandibular condyle from SAMP3 mice at $3(n=10)$, $6(n=10)$ and 9 months $(n=10)$ was evaluated using microcomputed tomography (micro-CT, $\mu \mathrm{CT}$ Comscan ScanXmate-RB090SS Tokyo, Japan) with the following machine specifications: $90 \mathrm{KV}, 89 \mu \mathrm{A}$, binning mode $(1220 \times 1216)$, projection 600, 10.869 micro meter/pixel. Each condyle was dissected and analyzed for the degree of trabecular bone marrow spacing.

\section{Results}

\section{Phenotypical changes of SAMP3 mice}

There were no significant changes in the body weight of mice between the experimental and control groups. In addition, there was no variation in the size of the condyle with or without mechanical loading.

\section{Histological changes of the TMJ condylar}

TMJ sections were stained with HE or Safranin-O green, Safranin-O is a cationic dye that binds to the negatively charged glycosaminoglycans (GAGs) to characterize the structural changes of the mandibular condyle in the experimental groups as compared to the control groups. At 3 months of age, the surface of the condylar cartilage in both groups was smooth and intact in HE staining, but the rates of positive Safranin-O staining was reduced 

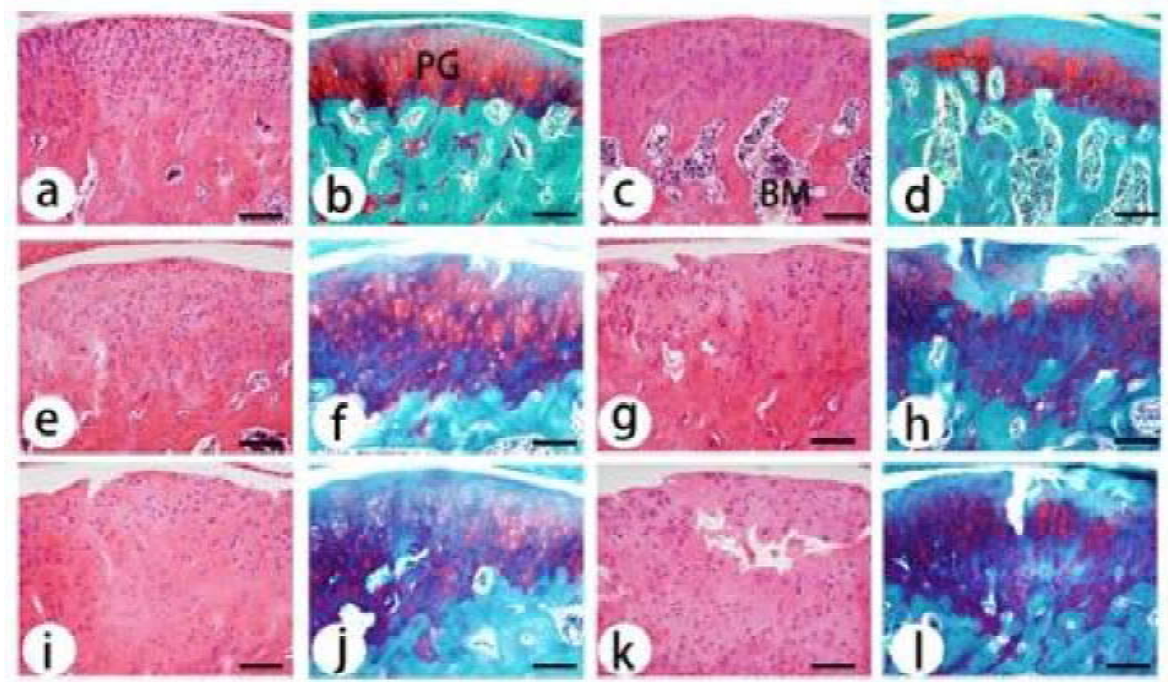

Figure 1: Parasagittal sections of the TMJ condyle in SAMP3 mice were stained with HE and Safranin-O green. (a and b) 3 month-old control; (c and d) 3 month-old experiment; (e and f) 6 month-old control; ( $\mathrm{g}$ and h) 6 month-old experiment; (I and j) 9 month-old control; (k and 1) 9 month-old experiment. Abbreviations: PG, proteoglycan; $\mathrm{BM}$, bone marrow. Bar $=100 \mu \mathrm{m}$.

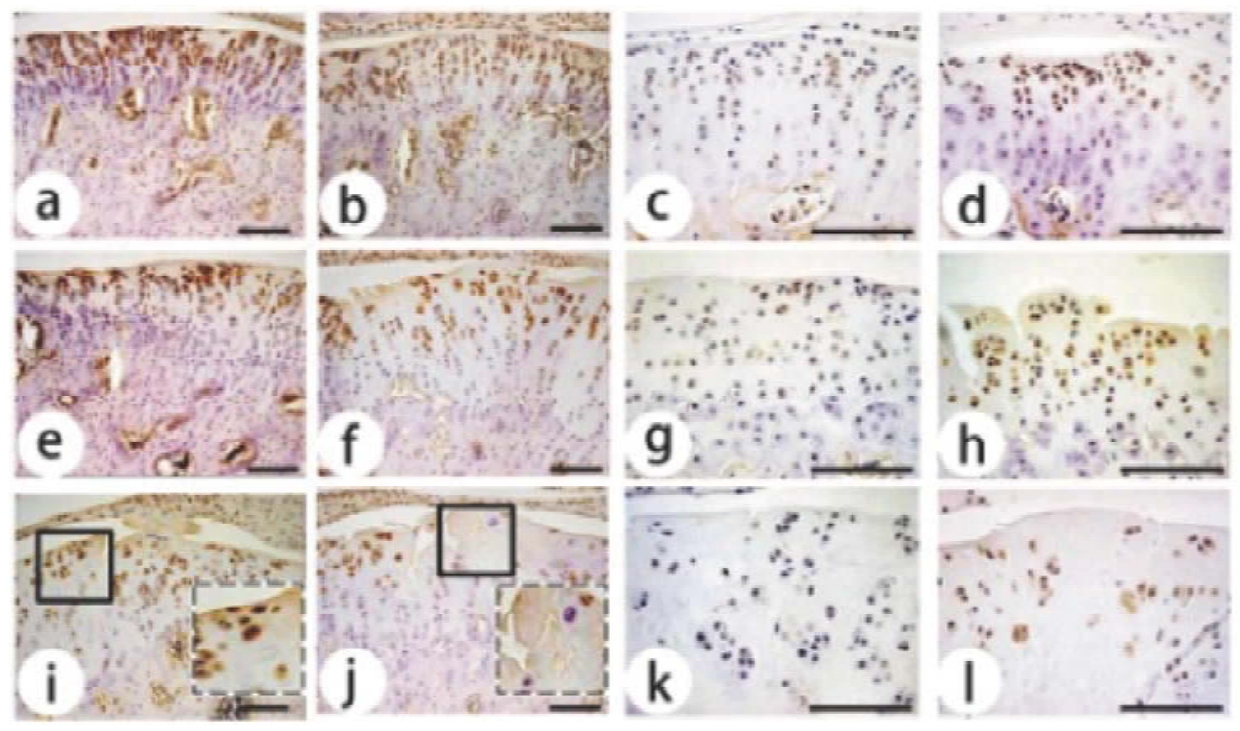

Figure 2: Type II collagen (a, b, e, f, i, j) and MMP-13 (c, d, g, h, k, l) immunostaining of the TMJ condyle in SAMP3 mice. (a and c) 3month-old control; (b and d) 3 month-old experiment; (e and g) 6 month-old control; (f and h) 6 month-old experiment; (i and k) 9 month-old control; (j and l) 9 month-old experiment. Boxed areas in (i) and (j) are shown at higher magnification in insets. Bar $=100 \mu \mathrm{m}$.

in the experimental groups as compared to the control groups (Fig. 1a-d). Also significant differences in the subchondral bone structure between the control and the experimental groups, with mechanical loading causing an increase in the trabecular bone marrow space (Fig. 1c and d). With progressive aging, the condylar surface with HE staining was eroded at 6 and 9 months, especially in the mice from the experimental groups. The proportion of positive Safranin-O staining also decreased, as expected, with 3 $>6>9$ months, respectively (Fig. 1e-1).
Degenerative changes and increasing subchondral ossification in the TMJ condylar cartilage tissue

\section{Immuohistochemistry for type II collagen and MMP-13}

The immunolocalization and perpendicular width of type II collagen positive chondrocytes layer was decreased in the experimental group with age dependent (Fig. 2b, f, j) compared to control condylar cartilage (Fig. 2a, e, f). This feature is consisted with a strong positive localization of MMP-13 (Fig. 2d, h, 1). However, control groups were negative for the expression for MMP-13 (Fig. 2c, g, k). 

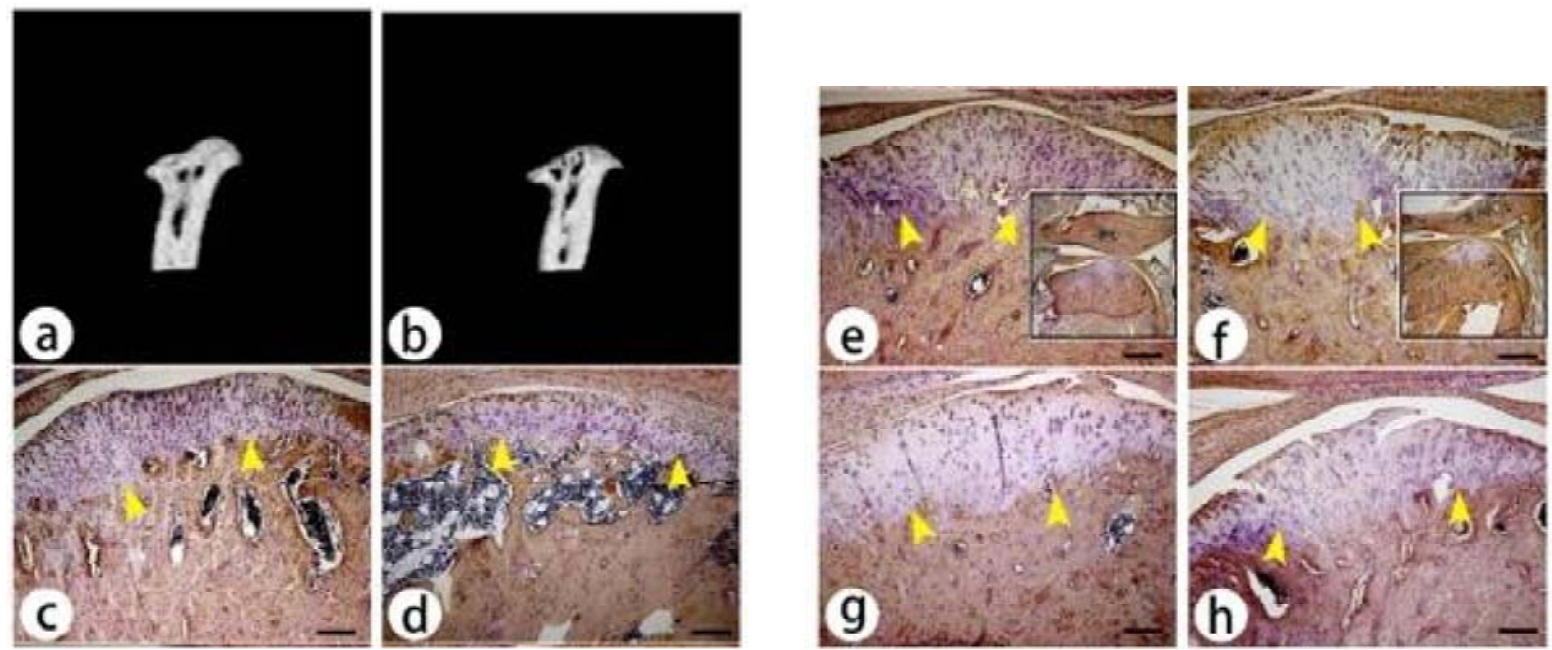

Figure 3: Subchondral ossification and type I collagen immunostaining of the TMJ condyle in SAMP3 mice. Representative images show mid-sagittal cross sections of the Micro-CT analysis of the TMJ condylar subchondral bone in 3 month-old SAMP3 mice control (a) and experiment (b); (c) 3 month-old control; (d) 3 month-old experiment; (e) 6 month-old control; (f) 6 month-old experiment; Lower magnification areas are shown in insets in (e) and (f); (g) 9 month-old control; (h) 9 month-old experiment. Yellow arrowheads are shown the interface between the bone and cartilage tissue. Bar $=200 \mu \mathrm{m}$.
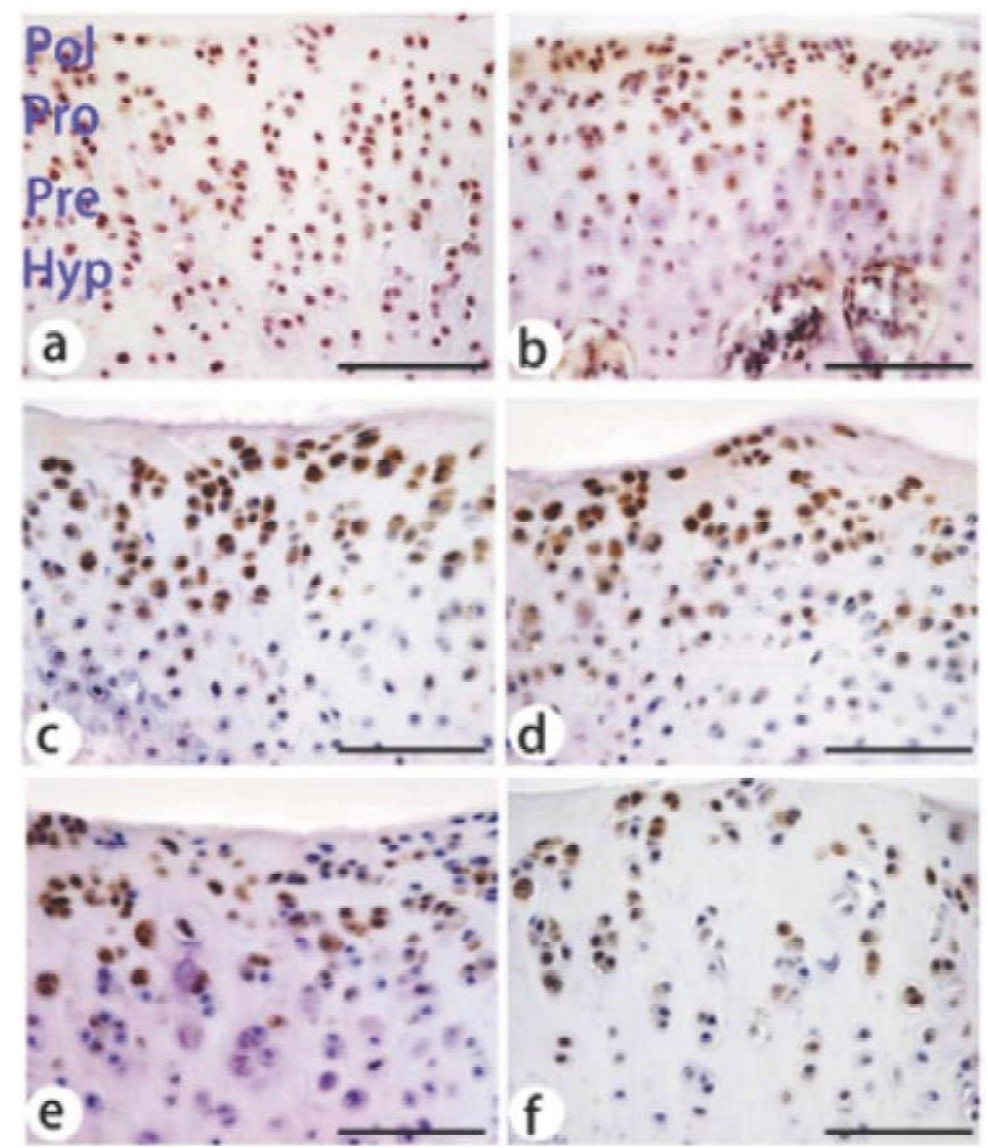

Figure 4: ß-catenin immunostaining of the TMJ condyle in SAMP3 mice. (a) 3month-old control; (b) 3 month-old experiment; (c) 6 month-old control; (d) 6 month-old experiment; (e) 9 month-old control; (f) 9 month-old experiment. Abbreviations: Pol, polymorphic cell layer; Pro, proliferative cell layer; Pre, prehypertrophic cell layer; Hyp, hypertrophic cell layer. Bar $=100 \mu \mathrm{m}$. 


\section{Subchondral ossification and type I collagen localization}

As shown in Figure 3a and b, Micro-CT analysis was performed to examine the role of mechanical loading in the micro-architecture of the condylar subchondral bone of the TMJ. At 3 months, the mechanical loading in the experimental group caused a significant increase of the bone marrow space, as compared to the control group. Continuous increase of type I collagen in matrix mineralization was revealed in the experimental groups, appearing as a thin belt running vertically toward the surface of the condyle at all ages (Fig. 3d, f, h). Overall, the findings in the present study indicate that mechanical loading in an aging background causes a decrease in the number of chondrocytes in the condyle and their disorganization within the tissue.

\section{Wnt/ß-catenin signaling}

To further investigate the regulatory role of $\mathrm{Wnt} / \beta$-catenin signaling, we next evaluated changes of $\beta$-catenin expression in articular chondrocytes. At 3 months, $\beta$-catenin was still expressed in both the control and experimental groups of the condylar cartilage chondrocytes (Fig. 4a, b). With progressive aging, there was a corresponding decrease in the nuclear expression of $\beta$-catenin (Fig. 4c-f).

\section{Discussion}

The present study showed strong correlations between occlusal disharmony caused by mechanical loading and aging in the induction of OA-like lesions. Aberrant mechanical loading is one of the etiological factors of TMJ degenerative disease by either trauma or altered mastication ${ }^{19}$. SAMP3 mice are characterized by the degeneration of the bone and cartilage tissue of the $\mathrm{TMJ}^{6}$. Creating minor disorders of occlusion and mastication by grinding the molars of these mice with accelerated senescence is a useful tool to evaluate the putative function of aging of the condyle in the TMJ. Age-related changes in the cartilage matrix are reported to contribute to the development of $\mathrm{OA}^{20,21}$. In the present study, deterioration and abrasion of the articular surface, which is thought to be a hallmark of $\mathrm{OA}^{22,23)}$, were found in SAMP3 mice at 6 and 9 months of age, the severity of which increased with mechanical loading.

Histologically, Safranin and fast green staining was observed in the condylar cartilage, signifying the presence of PGs at 3 months in the control group. However, in the experimental group at the same age, there was a reduction in PG expression in Safranin-O stained sections. Similar decreases in PG staining were observed at 6 and 9 months. Loss of PG expression has been reported as a critical event of OA progression ${ }^{24,25)}$. Taken together, these results suggest the onset of OA disease at 3 months in the experimental group of SAMP3.

In the subchondral bone, micro-CT analysis showed that mechanical loading by occlusal disharmony caused a significant increase in the bone marrow space compared to the control group at all ages. This phenomenon, which shows an increased bone marrow space and resorption of trabecular bone, also suggests manifestation of the early OA disease ${ }^{26)}$.

Remodeling of connective tissues, such as cartilage, is an integral feature of normal growth and development. Maintenance of cartilage involves both the removal and re-synthesis of structural proteins, such as collagen, and an imbalance in these pathways can lead to the degeneration of the joint cartilage, such as that which occurs in $\mathrm{OA}^{27)}$. A similar change was reported by $\mathrm{Xu}$ et al. ${ }^{28)}$, where alterations in the ECM of the mandibular condylar cartilage led to OA of the TMJ. To understand the mechanisms of cartilage degeneration in TMJ, we investigated the expression patterns of the ECM constituents - collagens type I, II and MMP13 - in the condylar cartilage tissue. We found a decrease in the expression of type II collagen in the articular cartilage of the TMJ at all experimental age groups, as compared to their control counterparts. The chondrocytes, which play an important role in the production of a cartilaginous matrix ${ }^{29)}$, were disorganized, and the tissue displayed a decreased number of cells in response to both the mechanical loading and the increasing age of the mice. Furthermore, increased expression of MMP-13 coincided with a decreased expression of type II collagen. Thus, increased MMP13 expression may represent a key characteristic of cartilage degradation in SAMP3 mice.

Immunohistochemical analyses demonstrated an increase of type I collagen at 3, 6, and 9 months of age in the experimental groups, and showed progressive ossification of the cartilage tissue in a vertical orientation to the surface of the condyle. These findings closely align with another report that $\mathrm{OA}$ is associated with accelerating endochondral ossification ${ }^{30}$.

Wnt/ $\beta$-catenin plays an important role in the articular chondrocyte function, as well as in cartilage development at the pre-hypertrophic zone ${ }^{31,32)}$. Therefore, we examined $\beta$-catenin expression in both control and experimental groups. At 6 and 9 months, the nuclear expression of $\beta$-catenin was limited and decreased, respectively. This is especially important for the mice in the experimental group, where it is considered that the cartilage tissue can no longer regenerate.

Overall, the findings of this study indicate that induced occlusal disharmony in an aging background of SAMP3 mice promotes the TMD and the onset of OA, accompanied by alterations to components of the ECM and Wnt/ $\beta$-catenin signaling. This disorder contributes to irreversible cartilage tissue damage, and the findings presented here may provide insight into mechanisms to help reduce the manifestation of OA in the TMJ.

\section{References}

1. Harada H, Hasegawa S, Yamada S, Nishi H and Tamura Y. 
Epidemiological study of the TMD incidence in children and adolescents. J Jpn Soc TMJ 14: 179-183, 2002

2. Harada H, Hasegawa S and Tamura Y. Epidemiological study for longitudinal changes of TMD syndromes in children and adolescents. J Jpn Soc TMJ 16: 185-190, 2004

3. Martin JA and Buckwalter JA. Aging, articular cartilage chondrocyte senescence and osteoarthritis. Biogerontology 3: 257-264, 2002

4. Chen J, Sorensen KP, Gupta T, Kilts T and Yong M S. Altered functional loading causes differential effects in the subchondral bone and condylar cartilage in the temporomandibular joint from young mice. Osteoarthritis Cartilage 17: 354-361, 2009

5. Takeda T, Hosokawa M, Takeshita S, Irino M, Higuchi K, Matsushita T, Yumiko Tomita, Kimio Yasuhira, Hajime Hamamoto, Katsuji Shimizu, Masaharu Ishii and Takao Yamamuro. A new murine model of accelerated senescence. Mech Ageing Dev 17: 183-194, 1981

6. Chen WH, Hosokawa M, Tsuboyama T, Ono T, Lizuka T and Takeda T. Age-related changes in the temporomandibular joint of the senescence accelerated mouse. SAMP3 as a new murine model of degenerative joint disease. Am J Pathol 135: 379-385, 1989

7. Wadhwa S, Embree MC, Kilts T, Young MF and Ameye LG. Accelerated osteoarthritis in the temporomandibular joint of biglycan/fibromodulin double-deficient mice. Osteoarthritis Cartilage 13: 817-827, 2005

8. Chen J, Gupta T, Barasz JA, Kalajzic Z, Yeh WC, Drissi H, Hand AR and Wadhwa S. Analysis of microarchitectural changes in a mouse temporomandibular joint osteoarthritis model. Arch Oral Bio 54: 1091-1098, 2009

9. Teramoto M, Kaneko S, Shibata S, Yanagishita M and Soma $\mathrm{K}$. Effect of compressive forces on extracellular matrix in rat mandibular condylar cartilage. J Bone Miner Metab 21: 276286, 2003

10. Reynolds JJ. Collagenases and tissue inhibitors of metalloproteinases: A functional balance in tissue degradation. Oral Dis 2:70-76, 1996

11. Birkedal-Hansen H, Moore WG, Bodden MK, Windsor LJ, Birkedal-Hansen B, DeCarlo A and Engler JA. Matrix metalloproteinases: A review. Crit Rev Oral Biol Med 4: 197-250, 1993

12. Ohkubo K, Shimokawa H, Ogawa T, Suzuki S, Fukada K, Ohya K and Ohyama K. Immunohistochemical localization of matrix metalloproteinase 13 (MMP-13) in mouse mandibular condylar cartilage. J Med Dent Sci 50: 203-211, 2003

13. Jee W.S.S.: The skeletal tissues. In: Cell and Tissue Biology, 6th ed. ed by Weiss L, Urban and Schwarzenberg, Baltimore, 1988, pp 211-254.
14. Morris NP, Keene DR and Horton WA. Morphology and chemical composition of connective tissue: Cartilage. Connective Tissue and its Heritable Disorders. Wiley-Liss, New York, 1993

15. Nusse R, and Varmus HE. Wnt Genes. Cell press 69: 10731087,1992

16. Nagayama M, Iwamoto M, Hargett A, Kamiya N, Tamamura Y, Young B, Morrison T, Takeuchi H, Pacifici M, EnomotoIwamoto $\mathrm{M}$ and Koyama E. Wnt/beta-catenin signaling regulates cranial base development and growth. J Dent Res 87: 244-249, 2008

17. Hall BK. Bone and Cartilage Developmental and Evolutionary Skeletal Biology. $1^{\text {st }}$ ed. Elsevier Academic press, Sandiego California, 2005

18. Alcaraz MJ, Megias J, Garacia-Arnandis I, Clerigues V and Guillen MI. New molecular targets for the treatment of osteoarthritis. Biochem Pharmacol 80: 13-21, 2010

19. Milam SB. Pathogenesis of degenerative temporomandibular joint arthritides. Odontology. 93: 7-15, 2005

20. Grushko G, Schneiderman R and Maroudas A. Some biochemical and biophysical parameters for the study of the pathogenesis of osteoarthritis: A comparison between the processes of ageing and degeneration in human hip cartilage. Connect Tissue Res 19: 149-176, 1989

21. Loeser RF. Aging and osteoarthritis: the role of chondrocyte senescence and aging changes in the cartilage matrix. Osteoarthritis Cartilage 17: 971-979, 2009

22. Franklin CD. Pathology of the temporomandibular joint. Current Diagnostic Pathology 12: 31-39, 2006

23. Malemud CJ and Martel-Pelletier JP. Degradation of extracellular matrix in osteoarthritis: 4 fundamental questions. J Rheumatol 14: 20-22, 1987

24. Nagase $H$ and Kashiwagi M. Aggrecanases and cartilage matrix degradation. Arthritis research and Therapy 5: 94103, 2003

25. Pearle AD, Warren RF and Rodeo SA. Basic science of articular cartilage and osteoarthritis. Clin Sports Med 24: 1-12, 2005

26. Martel-Pelletier J., Lajeunesse D., Reboul P., Pelletier J.P.: The role of subchondral bone in osteoarthritis. In: Osteoarthritis: A Companion to Rheumatology. 1st ed. ed by Sharma L and Beenbaum F, Mosby, Philadelphia, 2007, pp 15-32.

27. Tchetina EV. Developmental mechanisms in articular cartilage degradation in osteoarthritis. Arthritis 2011: 1-16, 2011

28. Xu L, Polur I, Lim C, Servais JM, Dobeck J, Li Y and Olsen BR. Early-onset osteoarthritis of mouse temporomandibular joint induced by partial discectomy. Osteoarthritis Cartilage 17: 917-922, 2009

29. Garant PR. Cartilage and temporomandibular joint. In: Oral cells and tissue. ed by Dickson A. Quintessence, Carol stream, 
Abir Elshawi et al.: TMJ Degenerative Change in SAMP3 Mice

Illinois, 2003, pp 321-337

30. Ea HK, Nguyen C, Bazin D, Bianchi A, Guicheux J, Reboul $\mathrm{P}$, Daudon $\mathrm{M}$ and Liote, F. Articular cartilage calcification in osteoarthritis insights into crystal induced stress. Arthritis and Rheumatism 63: 10-18, 2011

31. Akiyama H, Jon P. Lyons, Akiyama YM,Xiaohong Y, Zhang R , Zhang Z, Jian Min Deng, Makoto M. Taketo, Nakamura
T, Richard R. Behringer and Pierre D. McCrea. Interaction between Sox 9 and beta-catenin control chondrocyte differentiation. Genes Dev 18: 1072-1087, 2004

32. Koike H, Ejiri S, Hanada K and Ozawa H. Age-related histological changes in rat mandibular condyle. J Bone Miner Metab 13: 10-16, 1995 
J.Hard Tissue Biology Vol. 21(4):399-406, 2012 
